\title{
On-Body Wireless Inertial Sensing Foot Control Applications
}

\author{
Lawrence Cheng \\ Computer Science \\ University College London, Malet Place \\ London, UK, WC1E 6BT \\ 1.cheng@cs.ucl.ac.uk
}

\author{
Stephen Hailes \\ Computer Science \\ University College London, Malet Place \\ London, UK, WC1E 6B \\ s.hailes@,cs.ucl.ac.uk
}

\begin{abstract}
In recent years, the use of inertial sensing for body motion recognition has been demonstrated. However, existing work generally focuses on upper-body movements, which involve smaller scale movements and are less rapid. In this paper, we present two distinctive types of demonstration that show how onbody wireless inertial sensing can be used to capture detail inertial information of the more rapidly moving lower-body segments (e.g. the foot). The first demonstration shows how useful coaching support information for a sprinting exercise are captured; the second demonstration shows how inertial information of the lower segments are used to support football computer game applications, through which the users may trigger the appropriate on-screen actions by their foot motion, instead of using the current hand-held inertial sensing controllers.
\end{abstract}

Keywords-action triggering; football; inertial sensing; sprinting; wireless sensors.

\section{INTRODUCTION}

The use of on-body wireless inertial sensors for distinguishing between different human body motions were demonstrated in existing work. For example, in [4][5][6], the use of accelerometers for monitoring everyday activities was presented. In [7], the martial arts movements of a subject are captured using multiple on-body wired inertial sensors. Common features of existing work are: a) they focus on using inertial sensors to capture inertial motion data of a subject for distinguishing between different types of motion; b) most demonstrations focus on less rapid motion (such as walking and jogging, upper-body movements, etc.).

In this paper, we will present the results of two distinctive demonstrations which show what wireless inertial sensors when mounted on foot - can achieve. Comparing to upperbody movements, lower segment movements are known to be a) much more rapid; b) involves much larger changes in acceleration (such as when shooting a football); and c) most often involves large number of limited moments of ground contact $^{1}$ [3][4]. Our aim is to demonstrate that how different applications can benefit from inertial sensors that can pick up the smallest details of a motion. In the first demonstration, we will show that wireless inertial sensors are capable of

\footnotetext{
${ }^{1}$ For example, the total time of ground contact of the foot during a $100 \mathrm{~m}$ sprint would be limited; however, the number of ground contact (i.e. number of steps) could be frequent (depends on the stride length).
}

capturing the smallest details of a sprint motion, which are used to derive useful coaching support information (such as stride frequencies, stride length, etc.) for coaching applications. In the second demonstration, we will show how inertial sensors mounted on a foot are capable of enabling a much more realistic control method for football computer games, in contrast to existing systems such as $\mathrm{Wii}^{2}$.

This paper is organised as follow: firstly, the on-body wireless inertial sensor that we have implemented for use in the demonstration will be presented. Secondly, the experiments we have conducted will be presented and analysed. The paper ends with a conclusion and future work.

\section{THE SENSOR PROTOTYPE}

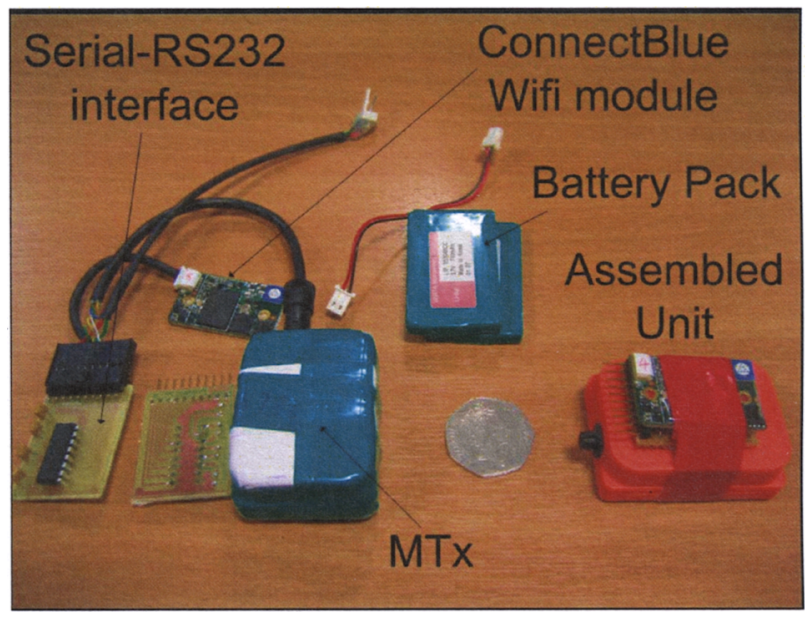

Figure 1 - The wireless inertial sensor prototype

To investigate the possibility of using inertial sensing on the lower-body segments for different foot motion monitoring applications, a set of experiments were carried out. Figure 1

${ }^{2}$ For example, in a sprinting game of WarioWare [1], the user must shake his/her hand-held control up and down rapidly to simulate a running player; in FIFA Soccer 2008 (the Wii version) [2], the user must swipe the hand-held controller accordingly to shoot a ball and tackle. Obviously, a more natural approach would be for the users to actually do the shooting and running, and the lower segment movements being captured directly by the sensors (and subsequently trigger the corresponding action on the screen. 
shows the wireless inertial sensor prototype that we have designed and implemented for these experiments. We chose the MTx sensor from xSens [9] as our sensing unit for its completeness: an MTx sample represents all essential (i.e. 6 Degree of Freedom) motion related data including acceleration, magnetometer readings, gyros readings, temperature, timestamps, etc. The sensor is configured to sample at its default rate (i.e. at $120 \mathrm{~Hz}$ ). It is connected to the UART of a connectBlue OWSPA311g 802.11 module through a RS232 transceiver. The sensor is powered by a pack of battery and would last for $1.5 \mathrm{hrs}$ when continually sampling and transmitting data wirelessly. The assembled unit weighs $\sim 75 \mathrm{~g}$ (including the battery pack). The sensor transmits data wirelessly to a remote data server (i.e. a laptop) using TCP, so all samples are gathered for analysis. Figure 2 shows the positioning of the sensor in the experiments (i.e. the right shank of the subject). This location is chosen because it has less fat hence provides a relatively more rigid surface (i.e. less vibrations means less noise). Note that, for the accelerometer, the front-back (i.e. forward) acceleration is $\mathrm{X}$, the up-down (i.e. vertical) acceleration is $Y$, whereas the horizontal acceleration is $Z$. The $3 \mathrm{D}$ gyro measures the rate of turn of a body segment along three planes (Figure 3).

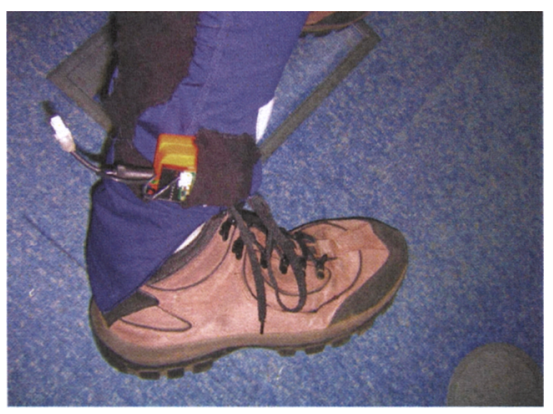

Figure 2 - An illustration of the position of the sensor (securely placed to a pocket of the right shank of the tracksuit)

b) Rotation around $Y$
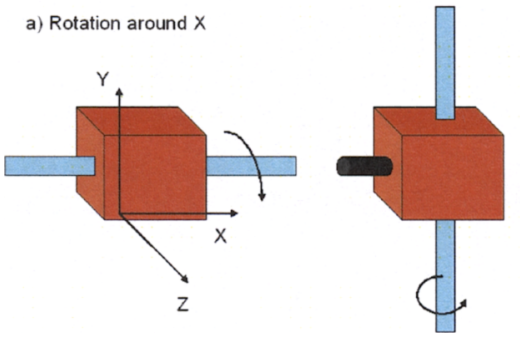

c) Rotation around Z

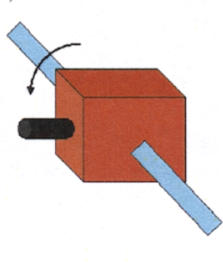

Figure $3-3 D$ rotation of a gyro

\section{INERTIAL SENSING FOR COACHING SUPPORT APPLICATIONS}

\section{A. Background}

In recent years, the possibility of using motion sensing systems for supporting sport's training has been under investigation [5][8][12]. In [8], it was discussed how athlete's motion data would be used for deducing useful coaching support information, such as stride frequencies, stride length, and more. In this section, we will demonstrate an on-foot inertial sensor can capture sufficient coaching support information.

\section{B. Results and Analysis}

Figure 4 and Figure 5 show the acceleration results when the sprinter sprints along the track, and walks back to the starting point. The results show that, the measured acceleration magnitudes during the sprinting session are much greater than the ones measured during the walking session; also, the vertical acceleration has the largest change in acceleration magnitude. The reason is that, the vertical and forward-backward planes are the planes of the body that are directly experiencing forces: when a step is made, the foot-on motion creates a vertical (and backward) force on the body; whereas the foot-off motion pushes the body forwards. There are relatively small forces being exerted on the body along the horizontal plane; although when the right foot takes off, the body is slightly tipped to the left.

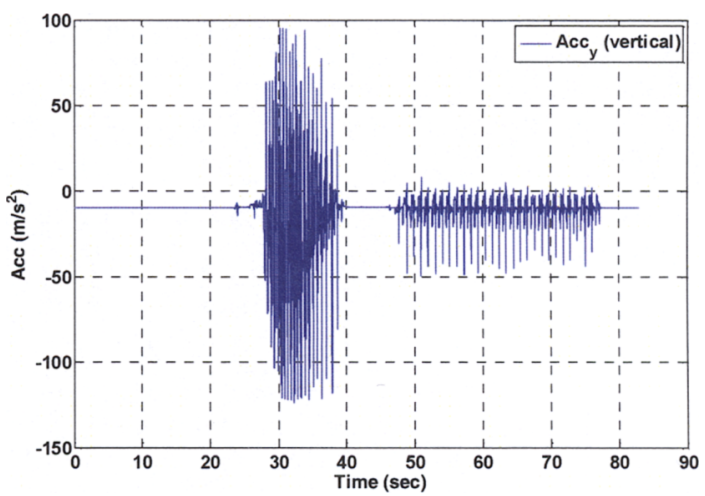

Figure 4 - Vertical acceleration (Y) of the sprinter

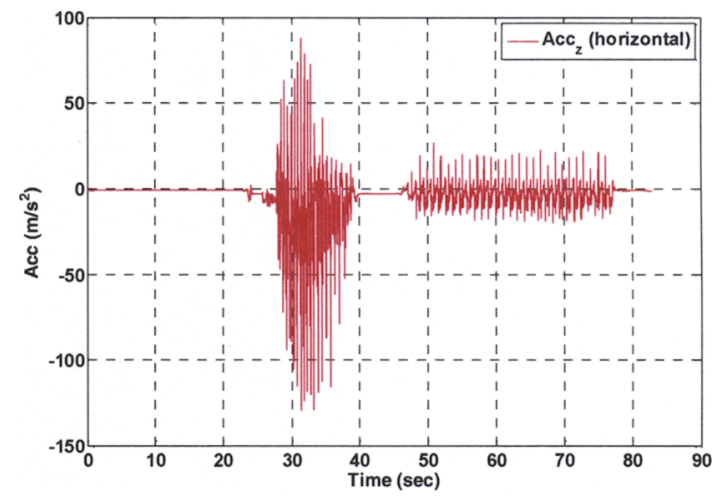

Figure 5 - Horizontal acceleration $(Z)$ of the sprinter

We chose vertical acceleration (i.e. $Y$ ) for signal differentiation because we are interested in determining the 
moments of the peaks (i.e. foot on and off) for the sprint and the walk; thus using the data of largest change of magnitude would provide us with the clearest differentiated signal. The differentiated signals [10] of the walking session and the sprinting session are shown in Figure 6 and Figure 7 respectively. By relating the sample number of the peaks of Figure 6 and Figure 7 to the time domain (i.e. Figure 4), we determined the average step duration for the sprint was $\sim 0.53$ sec; the step duration for the walk was $\sim 1 \mathrm{sec}$.

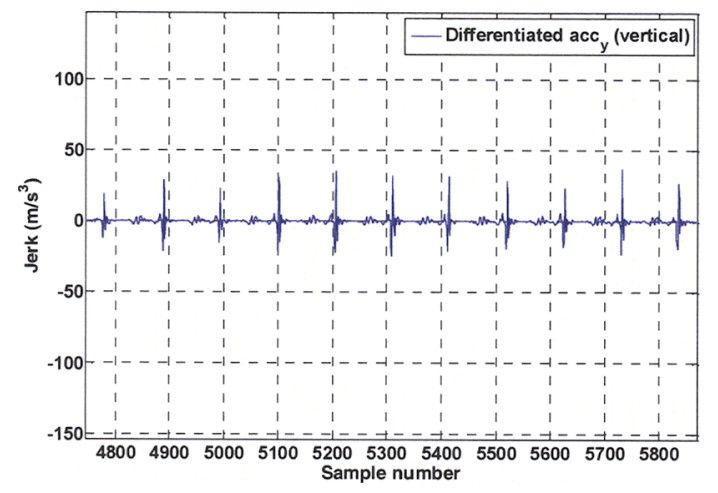

Figure 6 - Moments of peaks of the vertical acceleration during the walk (magnified version)

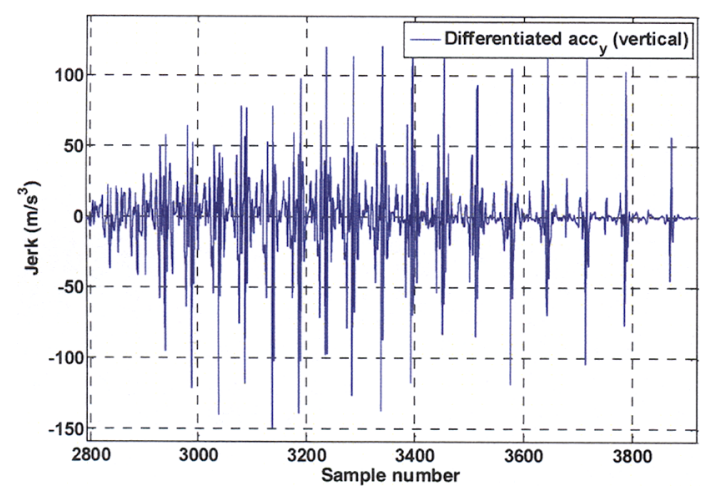

Figure 7-Moments of peaks of the vertical acceleration during the sprint (magnified version)

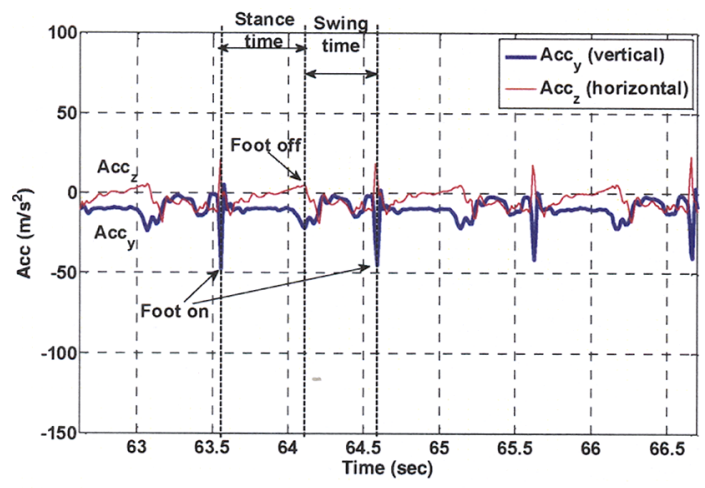

Figure 8 - Magnified version of a walking step

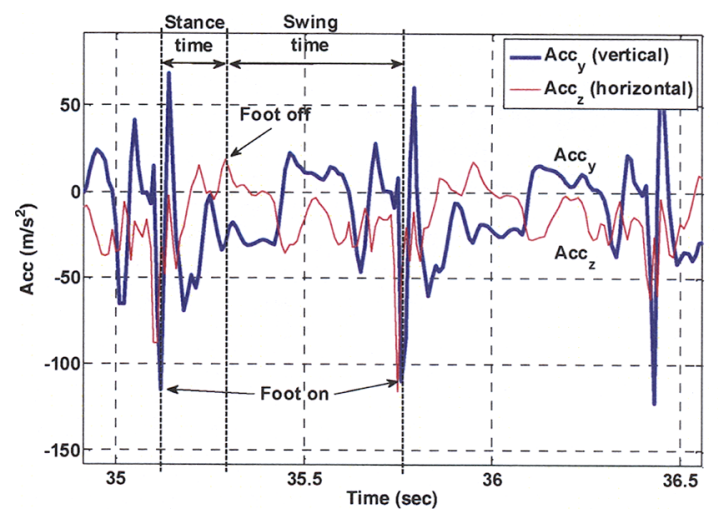

Figure 9 - Magnified version of a sprinting step

We have shown that some useful parameters, such as step duration, can be derived from the signals captured by inertial sensors. To show that inertial sensing is capable of picking up the smallest details of a sprint, further analysis was carried out using the results we have obtained. Figure 8 shows the magnified version of a walking step; the foot-on and foot-off moments were marked based on the foot analysis presented in $[11]^{3}$. We can clearly see that the stance time is approximately the same as the swing time. This means that the amount of time the foot is in contact with the ground is similar to the amount of time the foot is in the air (i.e. swinging). This is a typical behaviour of a walking step. Figure 9 shows the magnified version of a sprinting step: the stance time is only $1 / 2$ of the swing time. This result proves one of our earlier statements: that the total amount of time a professional sprinter's foot is on the ground is limited to achieve a maximum speed.

\section{InERTIAL SENSING FOR GAME CONTROL}

\section{A. Assumptions}

We have mentioned that our investigation focuses on rapid movements of the lower-body; thus, we assume the user is playing a football game, and is controlling a striker. A striker is chosen because his/her actions are most rapid; also, his role covers almost all types of on-the-pitch action: walking (e.g. when the ball is in his own team's territory, most often the striker is in the opposition's territory waiting for a pass), sprinting (e.g. running after the ball passed on through a long pass), and shooting. Note that these actions, currently, can only be activated by shaking a hand-held controller in the appropriate ways. In our initial experiments, the directions of shootings are also assumed to be controlled automatically by the computer (e.g. a shot would be made in the forward direction of the player). As a result, the user needs only to

${ }^{3}$ In brief, the sharpest peaks on the vertical plan indicate the moments of footon (i.e. the foot hits the ground and causes the body to suddenly change its acceleration); the peaks on the horizontal plan indicate foot-off moments because when the right foot pushes the body forward, hence the body swings to the left side. 
trigger the right action at the right time, and need not worry for the underlying complexity. However, we will present how directions are incorporated into control at a later stage, by showing how the system recognises side-passes. For simplicity, special game actions (e.g. "power shoot", "power run", goalkeeping etc.) are ignored; we will discuss later how these features could be included in a more advance design of the game controller. We assume that some predefined information is available in the system: each player of the team has a predefined profile that includes his name, club, age, etc. Such profile is extended to include useful parameters such as height, weight, etc. These information would be useful when computing the corresponding on-screen action triggered by the user's motion (see later).

\section{B. Results and Analysis}

A number of trials were carried out. Three types of motion were carried out in each trial: the first motion involved 10 walking steps ${ }^{4}$; the next motion was 10 running steps; then, a powerful shot was made at the end (Figure 10). This arrangement resembles the typical behaviour of a striker (as explained in last section). Note that - all motion was carried out at the same physical spot - this resembles a realistic scenario - people play video games in front of their TV. Thus, the results from this experiment would be different from those presented in section III. From the results (Figure 10), the walking steps are easily recognizable; however, the running steps and the shot were difficult to be distinguished from the $\mathrm{x}$ and $\mathrm{z}$ acceleration data. The vertical acceleration data, however, provides a much clearer signal. This is largely to do with the fact that, in game control, the body is moving at the same spot: the movements were not natural and there was literally no actual forward-backward motion, and the horizontal movements were restrained. However, the walking, running and shooting motion involves a relatively much larger degree of movements along the vertical plane (e.g. lifting the leg off the floor and into the air).
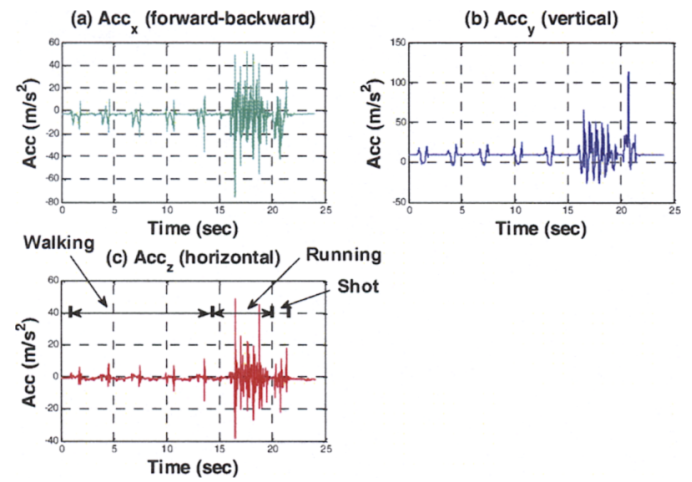

Figure $10-\mathrm{X}, \mathrm{Y}$ and $\mathrm{Z}$ acceleration data of a trial

\footnotetext{
${ }^{4}$ Note that, a step is made by two foot (i.e. right and left). Since the sensor is placed on the right foot, the inertial data would only show 5 "steps".
}

\section{Forward Shot Recognition using Gyros}

Although the shot is observable in the vertical acceleration data, its direction is unknown. Thus, in addition to accelerometers, we investigate the use of gyros in this circumstance. Figure $11 \mathrm{a}, \mathrm{b}$, and c shows the 3D gyros readings during the same trial respectively. Note that, the readings are referred to the rate of rotation around a specific axis.
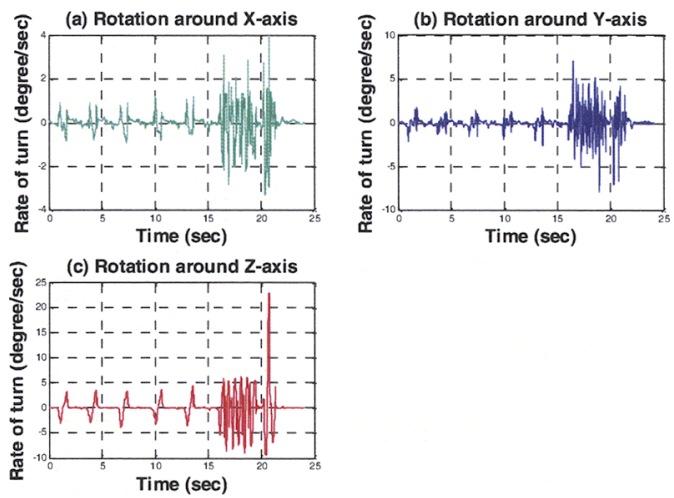

Figure $11-3 \mathrm{D}$ gyros readings during the trial

From the results (Figure 11), the rate of turn around the Zaxis is the clearest and sharpest; the shot can be easily identified from the running steps using the threshold analysis. The clearer signal along the Z-plane is because: when a shot is made by the right foot, the shank (i.e. lower right leg) is swung upwards (Figure 3c) much more rapidly than when a running step is made (a running step at the same spot essentially means the foot is lift up and down rapidly, which is a much smaller motion than swinging the foot upwards during a shot). Thus, the degree of rotation around the Z-axis (i.e. the foot swung forward) is the largest. In contrast, the degree of rotation around the $\mathrm{Y}$-axis and the $\mathrm{X}$-axis are relatively much smaller, and in fact, hard to distinguish from the running steps. Thus, in this case, the shot was in the direction of the body.

\section{Side-Passes Recognition}

In this section, we are interested in whether gyro readings can recognise the direction of a shot motion. Figure 12 shows the gyros readings for three left side passes made by the right foot during another trial (e.g. making a pass using the inside of the right foot). Side passes are completely different from shots in terms of their magnitude and direction. Note that, the average |rate of turn| is as follow: $|\mathrm{X}|>|\mathrm{Y}|$, and $|\mathrm{Z}|$ is much smaller than $|\mathrm{X}|$ and $|\mathrm{Y}|$. This result is in-line with a left-pass motion: the foot lifts and kicks to the left. The swing is around the $\mathrm{X}$-axis and is the largest because of the swing of the foot from ground to left upwards; the rotation around the Y-axis is the second largest because of the slight turn of the leg along the horizontal plane; in contrast to the forward shot analysed in section IV.C, the rotation around the Z-axis is the least because there is literally negligible forward-backward movement. For right-passes (i.e. passing using the outside of the right foot), the 
direction of the peaks would be in reversed order (due to the opposite direction of movement).
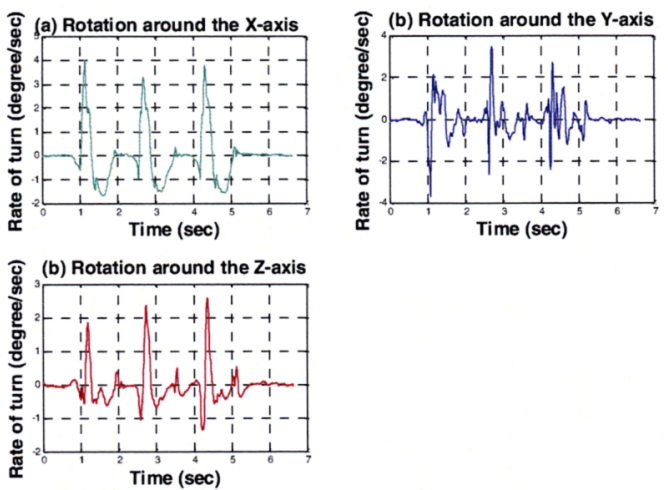

Figure 12 - Gyro readings for three left-passes by the right foot

\section{CONCLUSION}

Existing work have demonstrated how on-body wireless inertial sensing are applicable to upper-body motion recognition. In this paper, we have presented the results of two experiments which show wireless inertial sensing can also be used to capture lower-body segment motion which are much more rapid. In our first demonstration, we have shown that a wireless inertial sensing system that collects different types of inertial data from sprinters for coaching support. Our evaluation results show that vital coaching support information, such as step frequency, stance time and swing time, can be deduced from data collected by our system. Our second demonstration shows the use of inertial sensing of game control is not limited to monitoring upper-body movements. Using a football computer game as an example, we have discussed and demonstrated how inertial sensing can be used to distinguish between rapid lower segments movements, hence extending the range of games that can be supported.

\section{ACKNOWLEDGMENT}

Some of the work presented in this paper were undertaken in the context of the EPSRC-funded SEnsing for Sport And Managed Exercise (SESAME) project (EP/D076943).

\section{REFERENCES}

[1] WarioWare, http://www.warioware.biz

[2] FIFA Soccer 2008 (Wii): a review, http://review.zdnet.com/wiigames/fifa-soccer-08-wii/4505-9993 16-32371713.html

[3] K. Cocker, G. Cardon, I. Bourdeaudhuij, "Validity of the Inexpensive Stepping Meter in counting Steps in Free Living Conditions: a Pilot Study", in British Journal of Sports Medicine, 2006; 40:714-716.

[4] M. Makikawa, T. Isaka, T. Iida, S. Asajima, "Portable Jogging Monitor Device and its Application for Health Management", in Proceedings of IEEE EMBS Asian-Pacific Conference on Biomedical Engineering, Kyoto, Tokyo, Oct 2003, pp. 40-41.

[5] The SEnsing for Sports And Managed Exercise (SESAME) project, http://www. sesame.ucl.ac.uk

[6] T. Ahola, P. Korpinen, J. Rakkola, T. Ramo, J. Salminen, J. Savolainen, "Wearable FPGA Based Wireless Sensor Platform", in Proceedings of the $29^{\text {th }}$ Annual International Coference of IEEE EMBS, Lyon, France, August 3-26, 2007.

[7] E. Heinz, K. Kunze, M. Gruber, D. Bannach, P. Lukowicz, "Using Wearable Sensors for Real-time Regonition Tasks in Games of Martial Arts - An Initial Experiment", in Proceedings of the IEEE Symposium on Computational Intelligence and Games, Reno/Lake Tahoe, USA, May 2006.

[8] L. Cheng, S. Hailes, "An Experimental Study on a Motion Sensing System for Sports Training", short paper in the Proceedings of the $5^{\text {th }}$ European Confernece on Wireless Sensor Networks (EWSN), Bologna, Italy, Feb 2008

[9] The MTx sensor from xSens, http://www.xSens.com

[10] S. Nakazawa, T. Ishihara, H. Inooka, "Real-time Algorithms for Estimating Jerk Signals from Noisy Acceleration Data", in International Journal of Applied Electromagnetics and Mechanics, Vol. 18 (2003), pp 149-163.

[11] J. Lee, S. Cho, J. Lee, K. Lee, H. Yang, "Wearable Accelerometer System for Measuring the Temporal Parameters of Gait", in Proceedings of the $29^{\text {th }}$ Annual International Conference of the IEEE EMBS, Lyons, France, 23-26 August, 2007.

[12] L. Cheng, S. Hailes, "Managed Exercise Monitoring: A Novel Application of Wireless On-Body Inertial Sensing", in Proceedings of the $5^{\text {th }}$ IEEE International Workshop on Wearable and Implantable Body Sensor Networks (BSN), Hong Kong, June 2008. 